Meta

Journal des traducteurs

Translators' Journal

\title{
Entretien avec Lionel Salem
}

Aventures et mésaventures d'un dictionnaire des sciences

\section{François Gaudin}

Volume 40, numéro 2, juin 1995

Usages sociaux des termes : théories et terrains

URI : https://id.erudit.org/iderudit/002822ar

DOI : https://doi.org/10.7202/002822ar

Aller au sommaire du numéro

Éditeur(s)

Les Presses de l'Université de Montréal

ISSN

0026-0452 (imprimé)

1492-1421 (numérique)

Découvrir la revue

Citer ce document

Gaudin, F. (1995). Entretien avec Lionel Salem : aventures et mésaventures d'un dictionnaire des sciences. Meta, 40(2), 320-329.

https://doi.org/10.7202/002822ar

\section{Résumé de l'article}

Dans cet entretien, Lionel Salem présente Ia réalisation du Dictionnaire des sciences dont il a assuré la direction. Il expose ainsi les divers problèmes scientifiques et linguistiques qui se sont posés à lui. Il aborde également les difficultés réactionnelles et éditoriales d'une telle entreprise et les relations entre langue scientifique et langue commune.
Ce document est protégé par la loi sur le droit d'auteur. L'utilisation des services d'Érudit (y compris la reproduction) est assujettie à sa politique d'utilisation que vous pouvez consulter en ligne.

https://apropos.erudit.org/fr/usagers/politique-dutilisation/ 


\title{
ENTRETIEN AVEC LIONEL SALEM AVENTURES ET MÉSAVENTURES D'UN DICTIONNAIRE DES SCIENCES
}

Francolols Galldin

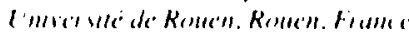

\begin{abstract}
Résumé

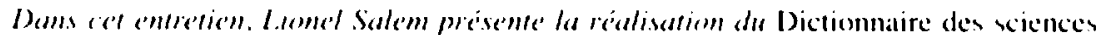

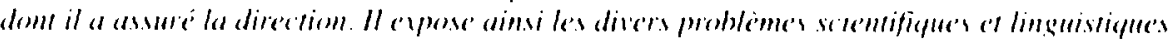

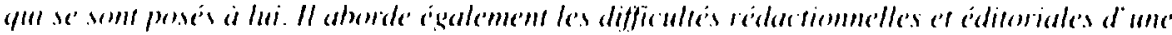

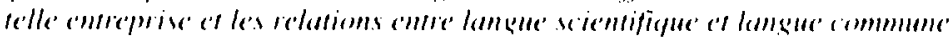

François Gaudin : Loms arez dirigé le Dictionnaire des sciences' paru chez Harhette.

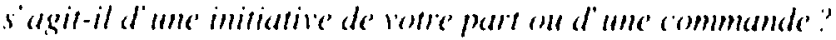

Lionel Salem : Dans le cas du Dictionnaire des sciences, contrairement à mes deux autres livres de vulgarisation parus, ee n'est pas une initiative de ma part. Je crois que Gérard Jorland, à l'époque conseiller che' Hachetle. est venu me voir un jour en me proposant. sinon de faire un dictionnaire des sciences. en tout cas. de faire quelque chose qui était asse/ semblable a ça et je lui ai dit : "Alors, pourquoi pas un dictionnaire?"

F.(i.: Et c'est vous qui arez rassemble les collaborateurs?"

L..S. : Je crois qu au début il voulait que je fasse quelque chose tout seul. Est-ce qu'il voulait que je fasse un livre de vulgarisation sur la science en général?" ou sur le sujet de la science? Je lui ai donc dit "Mais pourquoi ne pas faire un dictionnaire"?, et à ce moment-lì, il at dit "Mais alors vous feree tous les articles". J'ai répondu «Non, moi je veux bien coordonner. sinon trouver moi-même les collaborateurs” et ça s'est fait en deux temps. Dans le premier temps, jai contacté des gens qui étaient peu susceptibles de faire les articles, mais qui étaient très susceptibles de m' indiquer des collaborateurs, comme de Gennes?. Guinier' en physique. De Gennes, Guinier et bien d'autres m'ont donc indiqué des gens avec qui je pouvais travailler et qui ont eté des gens épattants. alors que d'atutres collegues, par exemple Jean (iénermont qui est un collègue d'Orsay, un kologiste, ont préféré faire les articles cux-mênes.

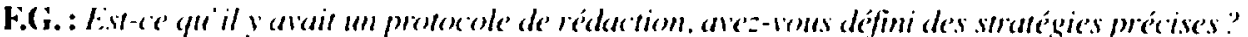
L.S.: Non. f̧a s'est fait d'une façon très impromptue el très désordonnée et je crois que ça se reflete d'ailleurs dans le livre lui-même qui a un côté un petit peu désordonné. Il y a dabord eu des délais énormes, en attendant qu'Hachetle veuille bien se décider sur le contrat. et ensuite sur le nombre de définitions et sur leur nature. Ensuite, après sêtre mis d'accord sur le chiffre de mille définitions, il fallait encore les choisir. Et ce choix a été très long. Je crois qu il a fallu six mois pour qu'on réunisse les gens compétents, et après fä. il a fallu faire signer les contrats à chatun des auteurs... Il y at eu pratiquement une année de perduce

Alors le résultat. c'est que ça s'est fait assè dans le désordre, et on s'est aperçu, une fois que les définitions élaient faites. qu'il y avait des redondances, qu il y avait des choses

Milu YI. 1009 
qui manquaient, il a fallu en refaire en catastrophe. Mais sur les 1020 définitions, il y en a tout de même $\mathbf{Y N O}$ qui étaient dans une liste initiale que j'avais concoctée avec les collègues. et ensuite il y en a eu 120 qui ont été un peu désordonnées...

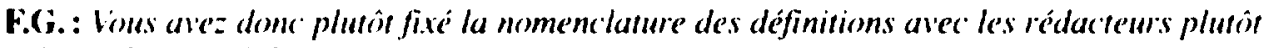
qui aver le comité de patromage?

1..S. : Ça dépendait. Par exemple, en mathématiques, j’ai d’abord fixé la liste avec JeanPierre Kahane, qui était au comité de patronage. et ensuite je l'ai peautinée avec Testard ${ }^{4}$ qui était le rédacteur. Guinier, je crois que je $n$ aa pas fait la liste avec lui, et pour certains domainess, la liste a été faite uniquement par les rédacteurs.

Il y a eu une grande réunion chez. Hachette où on a travaillé sur la liste. où. par exemple, il y avait des définitions de santé qu'on a carrément sabrées. On a décidé qu'on ne faisait pas «santé / médecine», donc on a fait une limite, très mal définie car vous verrez. qu'il y a des choses comme cervelet qui sont dans le dictionnaire on ne sait pas pourquoi. qui ont survécu à cette coupe.

F.G.: Comcernam l'étape de rédaction propreme'nt dite est-ce que vous alez quand même' défini une espèce de stratégie pour les définitions?

L..S.: Non. Cest-à-dire qu' Hachette a envoyé un papier disant: «ll faut une petite introduction et puis un truc principal, puis une petite conclusion": et, en fait, les auteurs ont peu suivi ça. Chaque auteur a mis sa patte propre à ses articles, ce yui a rendu ensuite l'homogénéisation très difficile.

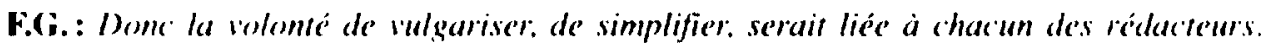
Vous n'arezas domné de consignes?

L..S.: Si, bien sûr! Il y avait une volonté de vulgariser que j ai exprimée personnellement à tous les rédacteurs, mais, entre la volonté que $j$ avais de vulgariser et ce que chaque rédacteur a hien voulu faire, il y a eu des écarts énormes et d'ailleurs, après la publication du dictionnaire, il y a des gens qui $m$ 'ont dit: «Regardez tel anticle, cest vraiment de la super vulgarisation et tel article c'est vraiment honteux. c'est vraiment un article de niveau DEA!» Donc, de ce point de vue-là, il ne faut pas non plus trop critiquer ce qu'on a fait soi-même, surtout vis-ii-vis des gens qui ont tait un énorme travail. Je dirais que, de ce côté-là, le dictionnaire n est pas un succès à cause justement de l'inégalité du niveau et de la qualité de vulgarisation.

F.(3.: Les ge'ns qui ont constitué l'équipe de rédacteurs étaient-ils tous an dépant motivés

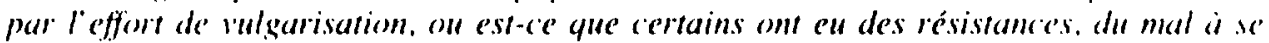
mertire dans la logiquar?'

L.S.: Ecoute\%. il y a eu trois cas de figure. Il y a des gens qui étaient des vulgarisalteurs nés. quelques-uns. rares. par exemple je crois une jeune chimiste (est-ce lsabelle Mallant?) qui a fait des définitions vraiment superbes de simplicité, toute seule sans qu'on lui apprenne. Il y en a d'autres à qui j'ai eu heau renvoyer trois ou quatre fois leurs articles à refaire, qui ne sont jamais arrivés à se mettre à niveau. Et puis, il y a le cas, beaucoup plus intéressant je trouve. de gens qui m’ont envoyé des articles extrêmement ésotériques. et avec qui j’ai passé une après-midi au téléphone. à qui $\mathrm{j}$ 'ai vraiment expliqué ce qu'il fallait faure en prenant un exemple avec eux. qui $m$ 'ont envoyé des choses beaucoup micux et qui devenus vraiment des vulgarisateurs de talent.

Je vais prendre un exemple. Frédéric Testard. I'homme qui a fail des définitions de maths. ("est lui qui a fait le plus de définitions, je crois qu"il y a eu 160 ou 120 détinitions de maths. ce qui représente un gros pourcentage. Au début. Testard m a sorti quelques définitions qui étaient dramatiques tellement elles étaient difficiles et ésotériques. Moi le test que jaai. c"est que si je ne comprends pas en tout cas personne ne va comprendre, non 
pas que je comprenne mieux, mais jaaime bien qu 'on $m$ 'explique les choses de façon très, élémentaire, quel que soit le domaine. J'ai passé une après-midi avec lui. il $\mathrm{m}$ a refait ensuite des définitions qui étaient presque loutes superbes et ensuite nous avons travaillé ensemble. nous avons fait ce livre qui s'appelle le's phas helles formule's mathématique's. Je crois qu'on peut dire maintenant que ce"est un vulgarisateur, mais il at appris à vulgariser.

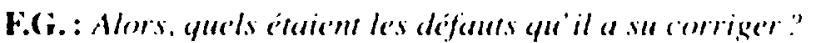

L.S.: Je crois vraiment qu une des premières choses, parmi celles qui me viennent à l'esprit comme ça dans le désordre. cest de ne pas introduire de mots compliqués. C"esta-dire ne pas introduire de mots comme l'espare $R$. ou quelque chose comme ca... Ou alors, si on a introduit l'éspace $R$. qui déjà est un concept difficile. on explique tout de suite la première fois ce que c'est. Donc, dans la mesure du possible, ne pas l'introduire et, s'il

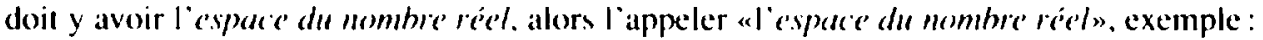
ce qu est un nombre réel. mais ne jamais lappeler $R$. Sinon. le lecteur ne va mème pas lire larricle, mais val le survoler des yeux. il val voir ee grand $R$ en caractere gothique, fal lui fera suffisamment peur et il ne va pas lire l'article.

Deuxiemement. je crois qu'il faut avoir une logique très imperturbable, c'est-à-dire que, dans l'explication. il ne faut pas qu'il y ait une faille qui fasse que l'on s'arrête. Fit ça. ce"ut peut-être une sorte de "don". entre guillemets. qu' on peut avoir personnellement. Je crois yue jeai é la chance, étant tout jeune, de ne pas comprendre les chosess sil n'y avail pas une logique très imperturbable. Ce qui fait que. quand je commençais ma thèse de chimie théorique. je lisais des articles de chimic théorique. je passais mon temps à aller voir mon patron ou des collègues de mon patron et à leur dire "Mor, je ne comprends pas la quatrieme ligne. alors on a dit ça. ça ça”. el on me disait "Efflectivement, là ça veut dire que...", "Effectivement, l'auteur s'est mal exprimé». C"était toujours ça.

Donc. ne pas avoir foi en la chose écrite et s'apercevoir que vraiment il faut que les choses soient expliquées, absolument... Donc 1) éviter l'ésotérisme. 2) avoir une logique très très dure el 3) bien sûr. respecter la vérité.

Len gens qui critiquent la vulgarisation, cest la première chose qu'ils disent: "Vous vulgarisez tellement que la verité scientifique est perdue." Je crois qu'on peut dire que. dans mon dictionnaire, on a respecté la vérité.

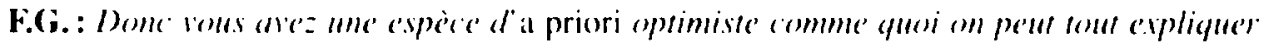
areec de's mots simples.

L.S.: Oui, “un a prioni optimiste», vous $m$ 'enlevez les mots de la bouche. je crois que je dirais qu'il n'y a pas de phénomène, si compliqué qu'il soit, qu' on ne puisse pas expliquer en une ou deux phrases. Je crois que. vraiment. même la notion de mécanique quantique. même les théories compliquées, tout de mème, je crois qu on peut les ramener à des idées simples qui. en une ou deux phrases, englobent pratiquement l'essentiel. Je crois que la logique humaine est tout de même quelque chose de très simple.

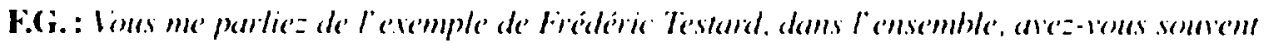
e'u besein de demander des récreriture's."

L.S.: Oh oui, jiai eu héatucoup de réécritures et j'ai éu même quelques ré-réecritures. J'ai tout lu deux fois.

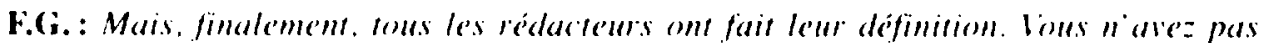
dio change' de collahorateurs.

1..S.: Non. Je ne sais même pas si j’en ai fait une seule moi-même.

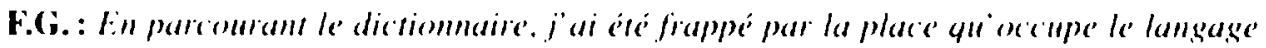

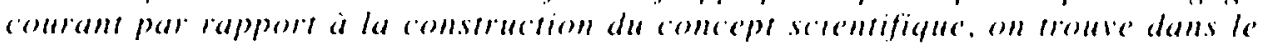


dictionnaire ume espece de confiance faite dans le langage commun. Mais selon vous. sa

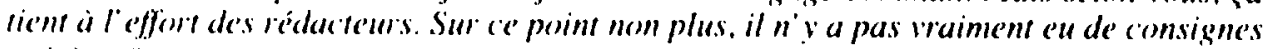
preicise's.

I..S.: Non. c'est probablement dû à moi, mais c'est dû aussi à Calherine Marquet ${ }^{5}$. de che\% Hachette. Je crois qu'il y avait deux lecteurs qui ont beaucoup revu de choses et il y a deux lectrices" qui mont aidé pendant tout un été et qui ont certainement contribué à cette homogénéisation.

F.(;.: Q Quel ćlait leur rôle'.

I..S. : Elles relisaient les articles pour que ce soit lisible pour des lecteurs de Hachette.

F.(i. : Apres rous:"

L.S.: Après moi. Une fois que c’était passé par moi, il y avait tout de même une partie "phraséologice en bon français" à faire. parce que je n'écris pas en très bon français.

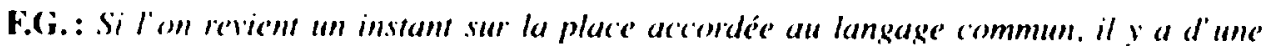
pait le fait que. pour definir un certain mombre de' concepts scienrifiques, on s'appuie sur de's motions commumes. Par ciemple pont supraconducteur, au lieu de partir directement sur supraconducteur. le rédac'felur parlé du métal. de ses propriétés de conductem et dans un

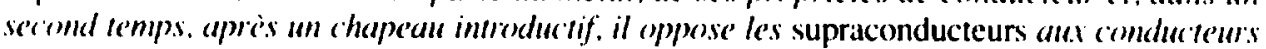
medinaire's que chacun commait. ("est un aspert. mais il ya a galement le fait quee, pour un certain nombre d'entrées. pas très important mais quand même. rous utilisez des notions commumes. des objots de la vie de lonis les jours, par exemple savon, détergent, brouillard. pour comstruire des motions chimiques ou physiques. Est-ce' que c'était un parti pris déli-

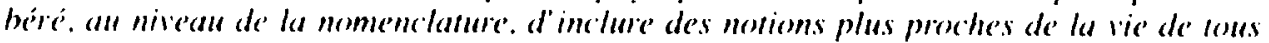
les jours que la nujorite des concepts scienrifiques?"

L..S.: Non, je ne crois pas. On a fixé la liste des définitions par consensus et je ne crois pas qu“il $y$ ait eu de parti pris en disant : "ll faut des mots du langage courant comme salon et des mots spécialisés comme supraconducteur."

F.G. : Certains rédacteurs ont rencomré des difficultés, vous l'avez dit. D'après rens, est-

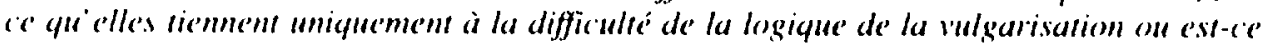
que sa depend des disciplimes.' Est-e quil y a des discipliness oi la mise on mots fiamsas est plus difficile."

I..S.: Je ne crois pas. Je crois simplement qu'il y a des gens qui ne se sont jamais mis dans la logique de vulgarisation. de même qu'il y a des gens qui ne sont pas pédagogues.

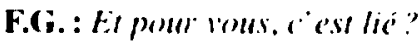

L..S.: Vous me le faites dire, mais je veux bien. Vous avez des chercheurs du CNRS qui somt incapables de faire un séminate correctement. parce qu'ils n'ont jamais appris à enseigner, et d'autres qui font des séminaires excellents parce qu'ils ont fá dans le sang.

F.(i.: Parmi vos rédacteurs, vous n' e'll avez jamais rencontré qui se méfiaient de la langue commume. qui. par exemple. civiteraient désordre pour parler d'entropie parce que. selon

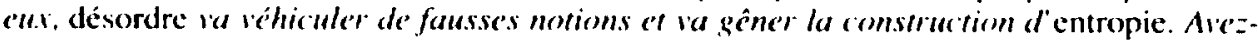

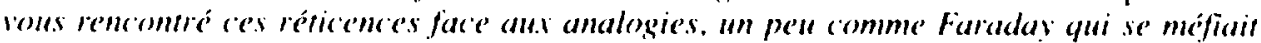
du mot courant pour désigne'r le phénemène électrique', alors qu' aujourd hui la métaphere' "l'st plus dis tout semsic...

I.S.: J'accepte l'attitude de quelqu'un qui fait une définition de l'entropie, qui dit "l'entropie est parfois comparée d'une façon relativement heureuse à la notion de désordr'. mais cette comparaison a des dangers parce que...". en donnant un exemple. "dans tel exemple, vous voyez bien que la notion de désordre et la notion d'entropie sont différentes». Bon. j’admets f̧a tout à fait. Mais ce que je n'admets pas, c'est tel ou tel scientifique 
qui est incapable de sortir des mols, de se libérer des mots scientifiques de son domaine. de sa discipline.

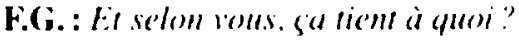

I..S. : Sa tient à une absence de maturité pédagogique, ça. ceest clair.

F. (i. : Ca ne liemt pas à la discipline?"

I..S. : Non. Pas une seconde. Pas plus d'ailleurs que pour la psychanalyse. Je suis convaincu que si lit prychanalyse sortait vraiment de celte gatngue yu est son langage. elle ne subirait pas l'échec qu elle subit mondialement... lises n"importe quelle revue de psychanalyse. cest absolument à tomber par terre... Ft on se moque des sorbonnards du XIIle siécle. mais fá dépasse de loin tout ee qu'on peut dire au XIIf' siécle!

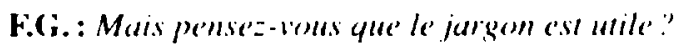

I..S.: Il y a quelques mots. je crois. On peut dire que la psychanalyse a amené quelques mots:

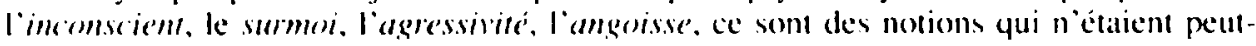
être pas définies avant. Tout le reste. je crois que c'est vraiment... le stmmot. c'est déjà un péu limite. mais tout le reste n était pas nécessaire.

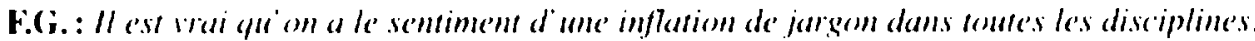

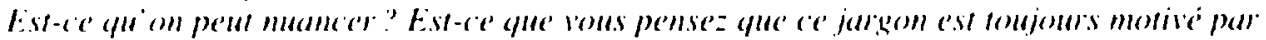

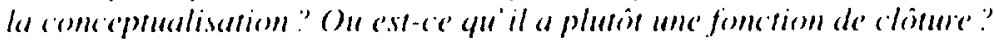

1..s.: Je crois qu’il peut avoir différentes fonctions. Il y al ceux qui mettent du jargon juste pour le plaisir de mettre du jargon, probablement. Moi, il mest arrivé d’introduire du jargon pour des raisons plus de "marketing". Jai toujours trouvé que si on avait un mot très frappant, percutante all point de vue umarketing du travail», fa aidait bealucoup.

Par exemple, on avait fait un travail. il y a quine ans, sur des réactions qui amenaient une molécule à la molécule image dans un miroir, et on avait appelé fä réduction marcissigur. Bon. javais trouvé ce mot et. finalement. les gens se sont polarisés sur le mot marissiqué. ils ont été très ohsédés par le mot, presque plus que par le contenu qui n'était pas d'une grande importance: et puis, it y a six mois. quelqu un a sorti un article en réemployant ce terme qui n'avait jamais été adopté.

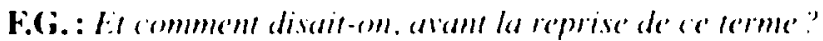

L..S.: (On utilisait d'autres termes moins parlants, comme cinamtiomérisation.

J'ai atussi trouvé un phénomène de photochimie quantique où. lorsque l'on pholoexcite une molécule qui a une double liaison qui se tord à 90 degrés. il y a une sorte de séparation de charge yui se produit: ça n'a jamais été vérifié expérimentalement. el j’ai appelé ça la polstisation sonduine. C"était vraiment somdain, f̧a se passait soudanement à un angle donné et le mot soudain avait un impact disons de "marketing". ou en fout cits attirait l"attention pour le lecteur et il y a eu de nouveatu une réaction très forte des chimistes dans le domaine, réaction au mot plus qu au contenu. À tel point que lorsque vous leur demandies "qu est-ce que c’est?". les gens avaient des définitions différentes, mais ils comaissaient bien le terme. (at mest donc arrivé une ou deux fois. mais ça a toujours été en me disant "Si jai vrament un phénomène nouveatu, je lui donne un nom qui essaie de frapper les gens".

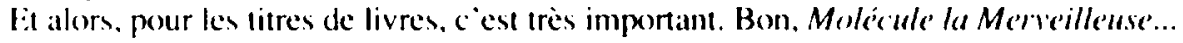
Les plus belle's formule's mathématiques, c'est peut-être plus commun, mais, par exemple. je suis en train de lerminer un livre de vulgarisation de génétique auquel a participé James Watson. I'homme de l'ADN ${ }^{7}$, on ne sait pas encore s'il va cosigner ou pas le livre, parce qu il y a peu participi. mais il y a eu beaucoup de discussions sur le titre, et jen avais trouvé un que je trouvais de nouveau percutant, qui était Mapping man. en français: afaisant la 
carte de l'homme", mais il $n^{\circ} y$ a pas l'allitération qu'il y a en anglais. Alors, l'éditeur américain a dit qu'on ne pouvait pas sortir un titre comme ça aux États-Unis parce que les mouvements de femmes... Ensuite, on a dit : Mapping man and woman, mais ça perdait de sa force. Alors évidemment en français, il n'y a rien. La carte humaine, je pense que c'est le titre qui serait le plus percutant, done ça faisait Human map ${ }^{\times} .$.

Mais finalement, je crois que ça sera lout de même publié en anglais sous le titre Mapping man parce que dans Mapping man. on a vraiment cette notion «on fait la carte de l'homme", qui ess d'ailleurs quelque chose d'assez extraordinaire comme idée. Il n'y a pas de mots nouveaux là-dedans, mais il y a tout de même l'adjonction de carlé et de homme'. de même qu'il y avait l'adjonction de molécule et de merveilleuse.

F.(i.: Il se troure que le laboratoire auquel j'appartiens travaille à l'élaboration d'un Dictionnaire des bio-industries. Le génic génétique, c'est tout de même un domaine à̀ il y a beaucoup de tormes anglais... Est-ce que vous ne rencontrerez pas de problemes poul. ingariser sa en fransais?

L.S.: Pour le moment, on a fait le livre en anglais. Ensuite, quand ça sera traduit, on verra. Je pense que là. on adoptera ce que les généticiens ont adopté, c'est-à-dire que s'ils ont adopté le terme anglais, on suivra. Je ne pense pas qu'on introduira de termes français nouveaux.

Ecoute\%. je vais prendre un exemple: je me suis fait faire une nouvelle carte de visite, j'ai mis fax dessus, et puis j'ai regardé la carte de visite de quelqu un qui a un poste analogue au mien au CNRS et j’ai vu que cette personne-là avait mis télécopie. Qu est-ce que vous voulez.? Effectivement, dans les annuaires de téléphone, on voit télécopio', mais pourtant je n'entends jamais personne dire télécopie, les gens disent far. Alors, il semble que là. la bataille est perdue pour tólócopice.

F.(i.: Oni. mais dans le domaine du génie génétique, il y a quand mèmé des conditions:

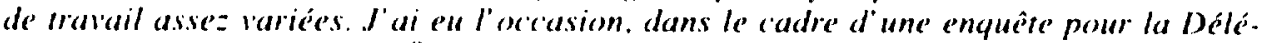
sation à la langue fransaise" de déponiller un certain nombre de revues, de participer à de's enguête's aupre's d'enseignants-cherchewrs, en fait, on s'apergoit qu' il y a massi-

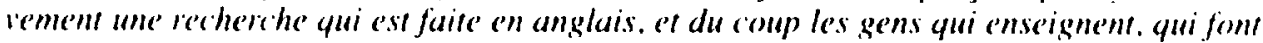

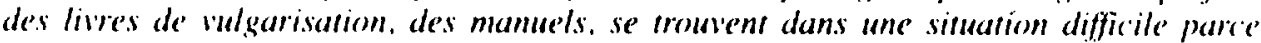

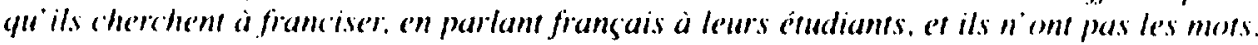
C"est pourquori on lrouse pas mal de solutions concurrentes, de synonymes.

Quesul vous dites spomanément "je ferai ce que ferom le's généticiens frangais". o'n

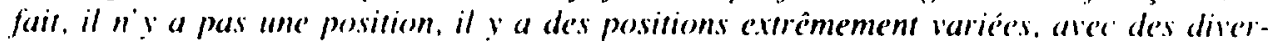
sences mème o'mtre dictionnaires. Il y a anssi un effort. qui est fait officielleme'nt: il y a des

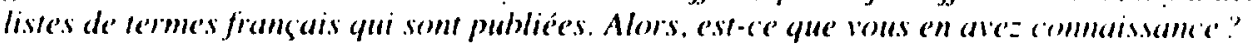
L.S.: Non, mais je ne sais pas. J'ai vu des listes, des listes types. C'est très difficile de savoir pourquoi un mot tient en français, pourquoi un mot ne lient pas. Par exemple, je crois que le mot legiciel tient bien pour le sofinare, et par contre je ne crois pas qu il y ait un mot pour le hard en français, les gens disent... Y'a un mot pour le hardware'? Ou les gens disent le hard"? Ils disent : "Tel logiciel... Le logiciel Word 4, elc."; par contre, ils diront "ll y a un problème de harduare" jai l'impression.

F.(i.: Moi. je n'entends pas hardware.

L..S. : C'est un peu comme la réforme de l'orthographe. Vous avez vu. la réforme de l'orthographe a capoté ! Pourquoi est-ce qu'elle a capoté? Au fond. parce qu'elle allait... Je crois que la réforme de l'orthographe, c'élait encore tout de même une réforme... |long silencé|. Je ne sais pas si la réforme de l'orthographe était vraiment une réforme de modernisation ou est-ce que c’élait une réforme. justement... plutôt de rendre encore plus français lè. choses françaises... Je ne sais pas, je ne sais pas... 
F.(i.: Non, c'étuit quand mêmé un effort de modernisation è c'était notamment déféndu par des gens qui socrupent de l' industrialisation du fransais.

1.S.: De toute façon. le français est presque une langue morte et, d'une certaine façon. je me disais «Si les Romains avaient essayé de modifier la façon d’écrire le latin au troisième ou au quatrième siècle après Jésus-Christ. ils n’auraient pas pu». Je crois qu'il faut laisser cette langue avec tout de même sa beauté... Justement. le fait que l"imparfait du subjonctif a un «âl» et tout, je crois qu 'il faut la laisser en l'état, et puis, quand elle n'a pas ce qu'il faut pour décrire des choses nouvelles...

Après tout, je crois lout de même que si vous ave\% une découverte française dans un domaine donné, vous aurez un mot français pour la chose. Si vous avez une découverte anglo-saxonne, vous aures le mot anglo-saxon. Alors on verra bien: si le monde anglosaxon fait $99 \%$ des découvertes, alors, les mots seront anglo-saxons. Et puis, vous aurez. un jour une découverte japonaise. vous aurez un mot japonais: mais si demain quelqu un trouve une nouvelle technique extraordinaire. par exemple. pour la reconnaissance de la parole... Pour te moment, il n'y a que des techniques assez pauvres, bâties sur les phonèmes, etc. Si quelqu un trouve en France quelque chose, il trouvera un mot. II peut inventer un mot français ou se servir d un vieux mot abandonné du langage français, il y en a heaucoup... Et à ce moment-là, les Américains l'adopteront. Il y a surprenamment de mots français dans le langage américain. vous savez.

F.(i. : Dams le demaine sciemtifique ?"

I..S.: Mais absolument, vous ave\%. par exemple. quelque chose en chimie qui s'appelle la compe dur roi. La conpe du rovi. cest une façon de couper une pomme en deux un peu compliquée: vous enfoncer le couteau puis vous le tournicoter. elc.. et vous coupez la pomme en deux morceaux qui sont tous les deux, on croirait que l'on aurait deux images. elles sont toutes les deux chirales, c'est-it-dire qu elles ne peuvent pas être superposées à leur image dans un miroir alors vous penseriez que vous auries une moitie qui serait une moitié gauche et une moitié droite. Et ce qui est très bizarre. cest que vous obteniez deux moitiés gauches. Bon, ca s'appelle la coupe du rom. Dans les articles de chimie dans le

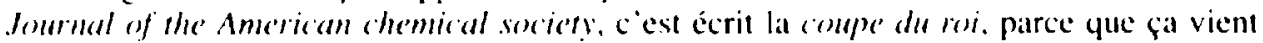
d'une vieille tradition paysanne française. Donc je crois qui on pourrail donner cet exemple. mais il y en a d’autres où le langage français est tout à fait utilisé.

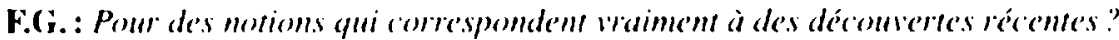

L.S.: Il y a des découvertes récentes où ils ont appliqué un terme du vieux français.

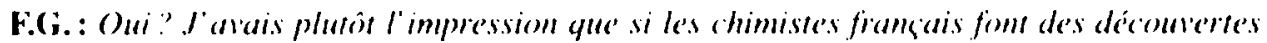
importantes, ils remt les public'r directeme'nt e'n anglais...

I..S. : Oui, ils peuvent utiliser un terme français....

F.(i.: lit sa sc fait?"

L.S.: Oui, par exemple. Jean-Marie Lehn qui a introduit. pour le genre de chimie qu il fait, le terme de chimie supra-moléculaire. C'est un mot qui est absolument abilingue", supra-moléculuire. ça marche aussi bien en français qu en anglais. Ce sont les meilleurs mots. D'ailleurs, lorsqu'on a cherché au début un titre pour le livre de génćtique. on a cherché un titre qui était...

F.(B.: Imerlingue"?

L..S.: Oui, interlingue, quand vous ave\% quelque chose qui est à la fois français et anglais. c'est évidemment le mieux. 
F.(3.: Alors, pour revenir à votre production éditoriale, dans Molécule la merveilleuse, it y a quand même quelque chose de significarif, c'est que vous supprime: un certain nombre de tormes techniques que rous remplacez par des mots à vous.

L..S.: Mais très peu. Une vingtaine.

F.G.: Oui. Pe'nsciz-vous que les mots utilisés habituellement constituent une espèce dobstarle pour le lecteur?

L.S. : Oui, un obstacle. Le mot va arrêter le lecteur, il serait trop difficile.

F.(i.: Et dans le Dictionnaire des sciences, vous êtes-vous posé le même problème?

L.S.: Oui, mais, sur 457 pages, finalement on baisse les bras.

F.G.: Et alors. y a-t-il cu des termes que bous avez été amenés à ne pas metrre én entrée. ou a mettre' sons d'autres rubriques?"

L..S.: Je ne $m$ 'en souviens pas. Je ne peux pas dire s'il y a eu vraiment consciemment un effort là-dessus. Dans le Dictionnaire des sciences, on n a pas fait consciemment le même effort que jai fait dans Molécule la merveilleuse qui est vraiment de traduire des mots. $J$ 'ai pu mettre de temps en temps sur la fiche d'un rédacteur. je $m$ 'en souviens maintenant. "Écoute\% est-ce qu'il n'y a pas un mot du langage courant qui remplacerait ça ?", mais je ne crois pas l'avoir fait de façon dirigée et régulière.

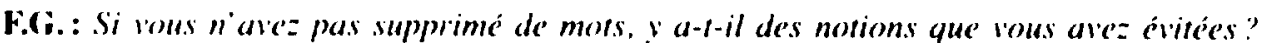
Par cxemple. il "n' a $n i$ matière, $n i$ antimatière, mais il y a antiparticule et particule élémentaire. Est-ce que cest volomtaire. sa?" Cola correspondait-il à des notions que vous rouliez civiter?'

I..S.: Non. Je ne sais pas. Si vous m’aviez demandé s'il y avait matière. j’aurais été surpris qu'il n'y soit pas. Il y a des choses qui ont été oubliées, par exemple, la théorie des distributions n'y était pas, je crois que ç'a été un oubli. Ç'a été rajouté dans le deuxième tirage. Il y a des choses carrément oubliées, involontairement ou parce que c’était trop récent: par exemple. je crois qu'on ne dit rien sur les ribozymes qui sont ces molécules d'ARN qui catalysent leurs propres réactions... Alors cạ. c'était très récent et ça n’a pas été mis. Et puis, il y a cu d autres cas où il y a eu des discussions, par exemple les découvertes de madame Le Doirin, vous savez. où elle fait des chimères : c'était de l'embryologie et il n'y avait pas aembryologie» dans le dictionnaire, done c'était limite. Alors on a discuté, ct tinalement, on a décidé de ne pas le mettre.

F.G.: Domc sa ne résultair pas d' une démarche disant si on met matière, sa véhicule des commotations méraphysique's, alors...

I..S.: Non. Je ne salis pas pourquoi on n'a pas mis matière, d ailleurs.

F.(i.: Ni anti-matière... lons incluez assez fréquemment dans votre ouvrage des éléments que je qualificrais de culturels qui somt soit des notations...

L..S.: Historiques! Oui, oui, ça, on me l’a demandé. C'est Hachette qui a beaucoup demandé.

F.G.: Mais vous, est-ce que vous pensez que c'est utile? Est-ce que vous pensez qu' un dictionnaire scientifique. à la limite d'un autre type. peut être également culturel?"

L.S.: Oui, plus ça va, plus je pense que ça devrait être généralisé. Il manque toujours des éléments historiques, et ensuite c'est très difficile de les trouver. Évidemment, il y a des histoires des sciences, mais ce n'est pas la même chose... Dans le mesure du possible, il y a tout de même eu une petite partie historique.

F.G.: Il y a même sourent. en plus de la simple mention de l'étymologie. une explicitation du rapport entre le nom et la chose. 
L.S. : Je crois que c'était une volonté presque plus de l'éditeur que de moi. Mais je crois que c’était une très bonne idée.

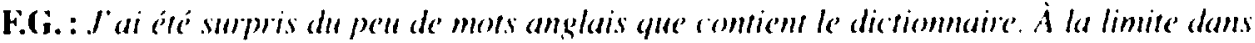

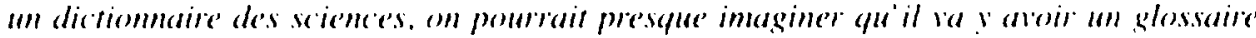
fransais-anglais...

L..S.: Non, ce n'était pas délibéré. Par contre, je regrelle que le dictionnaire n ait pas été traduit en anglais. L'éditeur aurait dû faire. dans le mois qui a suivi, un énorme effort pour essatyer de fatire les traductions; alors, c'est traduit en espagnol. c'est en train d'être traduit en italien. mais ça ne sera jamais traduit en anglais, ou alors ça sera peut-être repris un jour par un éditeur et refondu10.

Au début, on avait dit que ca s'appellerait Lés mille mots de la science et je pense que ça aurait éu une meilleure chance d'être traduit en anglais s il n'y avait pass éu l'idée de dictionnaire qui a toujours une connotation un peu encyclopédicue: vous prétendez. faire quelque chose d'un peu encyclopédique, et alors, vous tombez sur la critique de qui le lira, qui sera le rapporteur et qui dira qu il manque un tas de définitions importantes. Donc, je crois que si ça avait été lés mille mots de le scriencé, on aurait pu faire traduire.

F. (r. : Le mot dictionnaire forcuit un pe'u peur ".

L.S.: Ca n est pas que cal fait peur. cest que fal n est pas tout à fait un dictionnaire. Hachette a voulu appeler ça "dictionnaire" mais un dictionnaire de mille mots... je crois qu "il y a 7 (O)O mols. en fait, dans un bon dictionnaire scientifique.

F.(i. : Mais lis. c'ese un dictionnaire de hase'.

L.s.: Oui, cest-a-dire qu il y a tout de même $35(x)$ mots dans le lexique.

F.(i.: Oni. Parce quion a sonvent une présentation de plusieurs notions à la fonis. cer qui

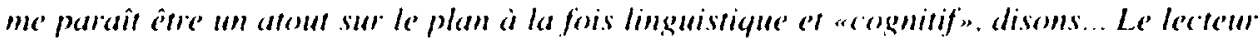

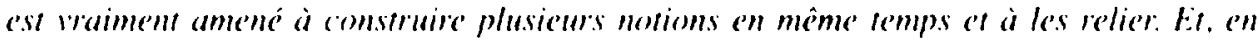
fait, on a tress rarement de monographies.

L..S. : Oui. Mais là. ce"st aux rédacteurs qu'il faut rendre hommage.

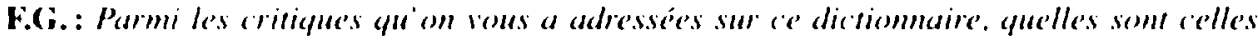
qui vouss ome imereresse".

L.S.: Je crois que la critique que jai entendue, à laquelle je suis le plus sensible moi-même. c'est l'inégalité de niveau. Ensuite, c'est que la couverture est très peu attrayante el que ça a certainement jouć. (a aurait pu se vendre très. très bien et il y a une eu une absence totale de promotion ches. Hachette... J'ai vu une publicité en latin, je crois, le 1.5 août ou quélque chose comme fal dans l.e' Monde'. Cest comme s"ils n'avaient pas voulu le promouvoir.

F.(i. : Eir même sans promotion?

1...S. : Il y a cu deux clubs qui en ont acheté 8000$)$ ou $9(0) 0$. Il y a cu un premier tirage de $20(0)$ qui a donc élé épuisé puisqu il a dô y en avoir par ailleurs $7(K()$ ou $8(K)$ qui ont ete vendus puis $2(K)$ distribues. Et puis, ils ont retiré $8(K)$ et a mon avis fá sera fini avec fal. Ça fera un total disons de $25(0)$ à $28(0)$, ce qui n'est pas mal. Mais Hachette. avec leur puissance de feu, si vraiment ils avaient fait une très belle couverture, s"ils avaient vraiment promu le livre au maximum et tout, ils auraient peut-être fait $50(0)$ ou 75 (KK).

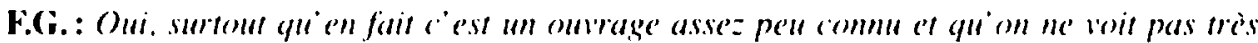
some'nt en bibliothèque".

L.S. : Je sais bien. Ft alors, ce qui est intéressant aussi, c'est que c'est le premier dictionnaire des sciences depuis... je crois que, depuis trente ans, il n'y avait pas eu un dictionnaire ou requivalent en France. 
F.(i.: Et save-rous s'il y en a un autre en chantier?

L.S.: Non. je ne pense pas. Et je déconseillerais à qui que ce soit de le faire!

Notes

I. Salem $\mid \varphi(x)$.

2. Pierre-(iilles de Gennes, prix Nobel.

3. Andre Guinier.

4. Frédéric Te'turd, coauteur avec Lionel Salem el Coralie Salem de l'ouvrage Les phes helles formules mathr. malnglues, voir références.

5. Responsable de l'éditıon.

6. Pascillinc Balland et Fabienne Joniaux.

7. Jamen Dewey Watson et Francis Crick ont élucidé la structure de l'ADN en 195.3.

8. Finalement. l'ouvrage a été publiés sous le titre $L$ homme' généticuté, voir Blocker et Salem (1994).

9. Contral du Giroupe de Recherches en Teminologie (URA CNRS 1164) atvec la Ixélégation générale à la langue frany̧asse sur l'impact des termes officiels dans ke domaine du génic génétique.

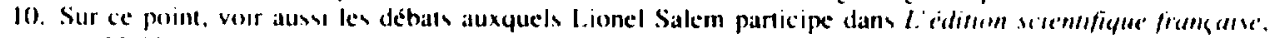
pp. 33.37

11. II n'a falt. al l'epoxque. I'objet d'aucune publicité dans les revues professionnelles destinées aux bibliothécaires.

\section{REFERENCES}

BL.OC KER. Ariel el Lionel SALEM ( $(494)$ : $L$ 'homme générique, postface d'A. Minkowski, Paris, Dunod. 320 p.

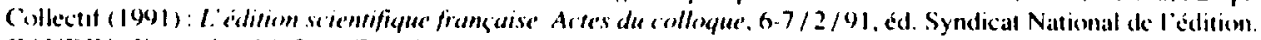
( $A$ IIDIN. Franģois (1992): "Terminologie et democratisation du savoir: a propes de dictionnaires scienti-

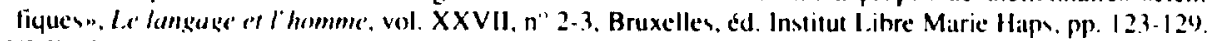

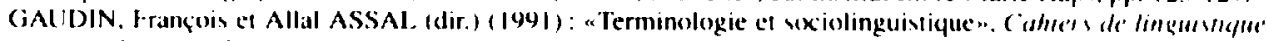
serwalc, n IX. Rousen. Université de Rouen. 213 p.

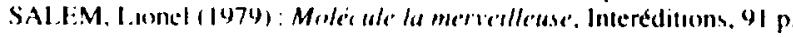

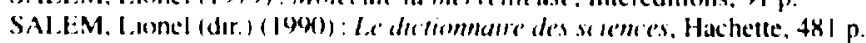

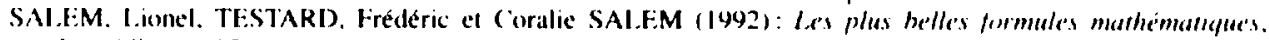
Interédittems, $151 \mathrm{p}$ 